\title{
Cost-Effectiveness Analysis of a HMGA2 Prognostic Test for Acute Myeloid Leukemia in a Canadian Setting
}

\author{
Gabriel Tremblay $^{1,7} \cdot$ Ben Rousseau² $\cdot$ Miriam Marquis $s^{3,4} \cdot$ Cyrielle Beaubois $^{3,4} \cdot$ Guy Sauvageau $^{3,4,5,6}$. \\ Josée Hébert ${ }^{3,4,5,6}$
}

Published online: 7 August 2019

(c) The Author(s) 2019

\begin{abstract}
Background Current strategies for risk stratification of patients with acute myeloid leukemia assign approximately $40 \%$ of patients to the intermediate-risk group, where uncertainty about optimal therapy still persists.

Objective The objective of this study was to assess the cost effectiveness of a HMGA2 prognostic test based on $H M G A 2^{+} / H M G A 2^{-}$expression, which improves genetic risk stratification in acute myeloid leukemia, and compare this test with the current standard of care in Canada.

Methods A cost-effectiveness model was developed from the Canadian National Healthcare Service and societal perspective using data from the Quebec Leukemia Cell Bank, published literature, and physician surveys. The model includes a lifetime horizon assessing the HMGA2 test vs. standard of care.

Results The HMGA2 test outperformed the standard of care at all time horizons culminating with estimated improvements of 1.92 and 3.12 months in leukemia-free survival and overall survival, respectively. Costs associated with the HMGA2 test were consistently lower, except diagnostic costs, routine medical costs, and costs related to infections and false positives. From a societal perspective, total lifetime costs were \$161,358 CAD and \$151,908 CAD with the standard of care and the HMGA2 test, respectively. The incremental quality-adjusted life-year gain was 0.138 , which led to dominance over the standard of care. Deterministic sensitivity analyses confirmed the results of the base-case scenario. Probabilistic sensitivity analyses revealed that for a willingness-to-pay threshold of $\$ 100,000 \mathrm{CAD}$, the probability of cost effectiveness was $87.19 \%$. Conclusions The HMGA2 test is estimated to improve leukemia-free survival and overall survival outcomes, and yield costs savings from a healthcare system and societal perspective.
\end{abstract}

\section{Introduction}

Acute myeloid leukemia (AML) is the fourth most common blood cancer in Canada [1]. In 2013, 1315 new cases of AML were diagnosed in Canada, approximately 3.8 cases per 100,000 people [1]. Furthermore, trends in the incidence

Electronic supplementary material The online version of this article (https://doi.org/10.1007/s40258-019-00503-5) contains supplementary material, which is available to authorized users.

Gabriel Tremblay

gabrielt.ecn@gmail.com

$\checkmark$ Josée Hébert

josee.hebert@umontreal.ca

Purple Squirrel Economics, New York, NY, USA

2 Adelphi Values, Bollington, UK

3 The Leucegene Project, Université de Montréal, Montreal, QC, Canada of AML indicate an increase with age from 0.3 to 6.5 cases per 100,000 persons in patients younger than 65 years old to 8.6 to 26.1 cases per 100,000 people in patients over 65 years old [1] and thus, a higher number of cases is expected in the aging Canadian population.

Accurate assessment of prognosis has become a key part of AML management. To ensure a better treatment response or avoid the risks of treatment resistance and treatmentrelated mortality, physicians are recommended to stratify

4 Quebec Leukemia Cell Bank, Research Centre, Maisonneuve-Rosemont Hospital, 5415 l'Assomption Blvd, Montreal, QC H1T 2M4, Canada

5 Division of Hematology-Oncology, Maisonneuve-Rosemont Hospital, Montreal, QC, Canada

6 Department of Medicine, Faculty of Medicine, Université de Montréal, Montreal, QC, Canada

7 Geneconomics Inc, 1372 rue Du crépuscule, Lévis, QC, Canada G7A 4K3 


\section{Key Points for Decision Makers}

Total lifetime costs associated with the HMGA2 prognostic test $(\$ 151,908 \mathrm{CAD})$ were lower than standard of care $(\$ 161,358 \mathrm{CAD})$. Probabilistic sensitivity analyses revealed that for a willingness-to-pay threshold of $\$ 100,000$ CAD, the probability of cost effectiveness was $87.19 \%$.

The HMGA2 prognostic test was estimated to improve clinical outcomes in acute myeloid leukemia. The analysis was conducted from a healthcare payer and societal perspective and showed the new test to be a dominant strategy, with a quality-adjusted life-year gain of 0.138 and a cost saving of \$9451 CAD.

patients based on genetic risk [2,3]. Among clinical factors, age, comorbidities, and performance status are considered important determinants of treatment outcomes and are generally used in guiding therapeutic decisions [4-6].

Although clinical factors have proven extremely useful in informing early decisions, cytogenetics (the study of chromosome abnormalities in leukemic cells) has been shown to be a strong prognostic factor of patient outcomes such as complete remission (CR), leukemia-free survival (LFS), and overall survival (OS) [7-9]. As such, patients with AML can be stratified into favorable (good survival), adverse (poor survival), and intermediate cytogenetic risk groups. Testing for NPM1, FLT3, and CEBPA gene mutations, and more recently screening for specific mutations associated with a poor prognosis (RUNX1, ASXL1, and TP53) are also recommended for genetic risk stratification [10]. Stratification based on cytogenetics and the presence or absence of these mutations has provided a means of better treatment decision making. The risk category guides the choice of consolidation therapies, which is straightforward for favorable (consolidation chemotherapy) and adverse (allogeneic hematopoietic stem cell transplantation [HSCT]) risk patients. However, up to $40 \%$ of patients with AML are still classified in the intermediate-risk group [8, 11, 12]. As a result, uncertainty persists concerning whether these patients should be treated with consolidation chemotherapy or assigned to HSCT after induction therapy.

The recent identification of a novel biomarker has led to advancements in the prognostication of a subgroup of patients with AML. The high-mobility group AT-hook 2 (HMGA2) marker was found to be expressed at high levels in approximately $22 \%$ of patients with AML [13]. A prognostic test based on an HMGA2 expression signature that can be used to stratify patients with AML was developed and validated in two independent sample cohorts of patients with
AML. Results of this study showed that the HMAG2 test identified patients who have poor clinical outcomes including a lower frequency of CR, worse LFS and OS, and added independent value to existing prognostic factors. This test also reclassified approximately $17 \%$ of intermediate-risk patients ( $\sim 7 \%$ of all patients with AML) into the poor-risk category, including patients who were negative for the six mutations detailed above. Moreover, $51.7 \%$ of adverse-risk patients (based on cytogenetics and poor-risk mutations) had a positive HMGA2 test $(\sim 12.6 \%$ of all patients with AML). These patients were unresponsive to standard intensive therapies [13].

In Canada, most patients with AML currently undergo diagnostic tests to detect cytogenetic abnormalities and $N P M 1, F L T 3$, and CEBPA mutations; however, mutational screening for RUNX1, ASXL1, and TP53 requires a sequencing platform that is not available in all molecular diagnostic laboratories. It is expected that a good proportion of these adverse-risk patients would be detected by testing for $H M G A 2$ expression because the HMGA2 test is positive in 27 of $45(60 \%)$ patients with mutations associated with a poor prognosis [13].

Given the current diagnostic paradigm, we assessed the cost effectiveness of using a HMGA2 prognostic test for patients with AML compared with standard of care, within the context of the Canadian National Health Service. Considering the low cost of this new test compared with the overall cost of the treatment pathway, we deemed it unlikely for the prognostic test to show a major financial burden for Canadian payers. Therefore, rather than conducting a cost analysis or budget impact analysis, we conducted a costeffectiveness analysis where we have assumed the new test would lead to changes in the treatment pathway as a result of additional information provided about the patients. The aim of the analysis was to provide decision makers with evidence of the impact of introducing a new prognostic test on the whole treatment pathway, rather than focusing solely on the cost of the test. The analysis was performed from a healthcare system perspective, as per Canadian Agency for Drugs and Technologies in Health guidelines in support of reimbursement, but also included a societal perspective presented as an additional scenario. The societal perspective also included indirect costs (friction costs), which can be an important factor for the active population.

\section{Materials and Methods}

We developed a partitioned survival model to evaluate the cost effectiveness of a prognostic test used to stratify adult patients with AML to treatment compared with the current standard of care, i.e., standard tests. The HMGA2 test diagnostic results used in this model were based on Marquis 
et al. [13]. This analysis excluded patients with acute promyelocytic leukemia because of differences in treatment and survival compared with other subtypes of AML. The model was developed in Microsoft Excel (Microsoft Corporation, Redmond, WA, USA).

\subsection{Model Description}

With a partitioned survival modeling approach, patients were assumed to be in one of three mutually exclusive health states at any given time: alive with no progression (LFS), alive with disease progression (relapse), or death (Fig. 1). Other modeling techniques, mainly Markov model and discrete-event simulation, were tested, but were deemed less optimal in the Canadian context. Based on the unique strength of the patient-level data provided by the Quebec Leukemia Cell Bank [13, 14], developing a model directly based on these data and outcomes for Canadian patients was considered stronger than relying on assumptions based on the literature. While a Markov model can also be based on patient-level data, a partition survival approach allows exact replication of the Kaplan-Meier survival curve for the different treatment pathways. The clinical validation confirmed that a model matching as closely as possible the Kaplan-Meier would be superior. The proportion of patients in each health state over time was estimated by fitting the Kaplan-Meier survival functions (partitions) for LFS and OS and calculating the area under the curve. The Kaplan-Meier survival functions were extrapolated after data cut-off to include a lifetime horizon starting at AML diagnosis using the best fitting parametric curve, i.e., using the exponential functional form (see Fig. S1 of the Electronic Supplementary Material [ESM]). This stepwise approach ensures the model fully utilizes the patient-level data and can also be extrapolated to a lifetime horizon. This is considered a more robust approach, which limits uncertainty before extrapolation cut-off as it relies on direct patient data. The best fitting extrapolation model was selected using literature-based criteria, such as a visual fitting, a statistical fitting such as an Akaike and Bayesian information criterion, realism of the extrapolation, and $\log -\log$ plots $[15,16]$.

The primary goal of the cost-effectiveness analysis was to estimate the expected LFS years, OS life-years (LYs), quality-adjusted life-years (QALYs), and lifetime treatments costs associated with adult patients with AML using the test (i.e., remission induction and consolidation rounds of
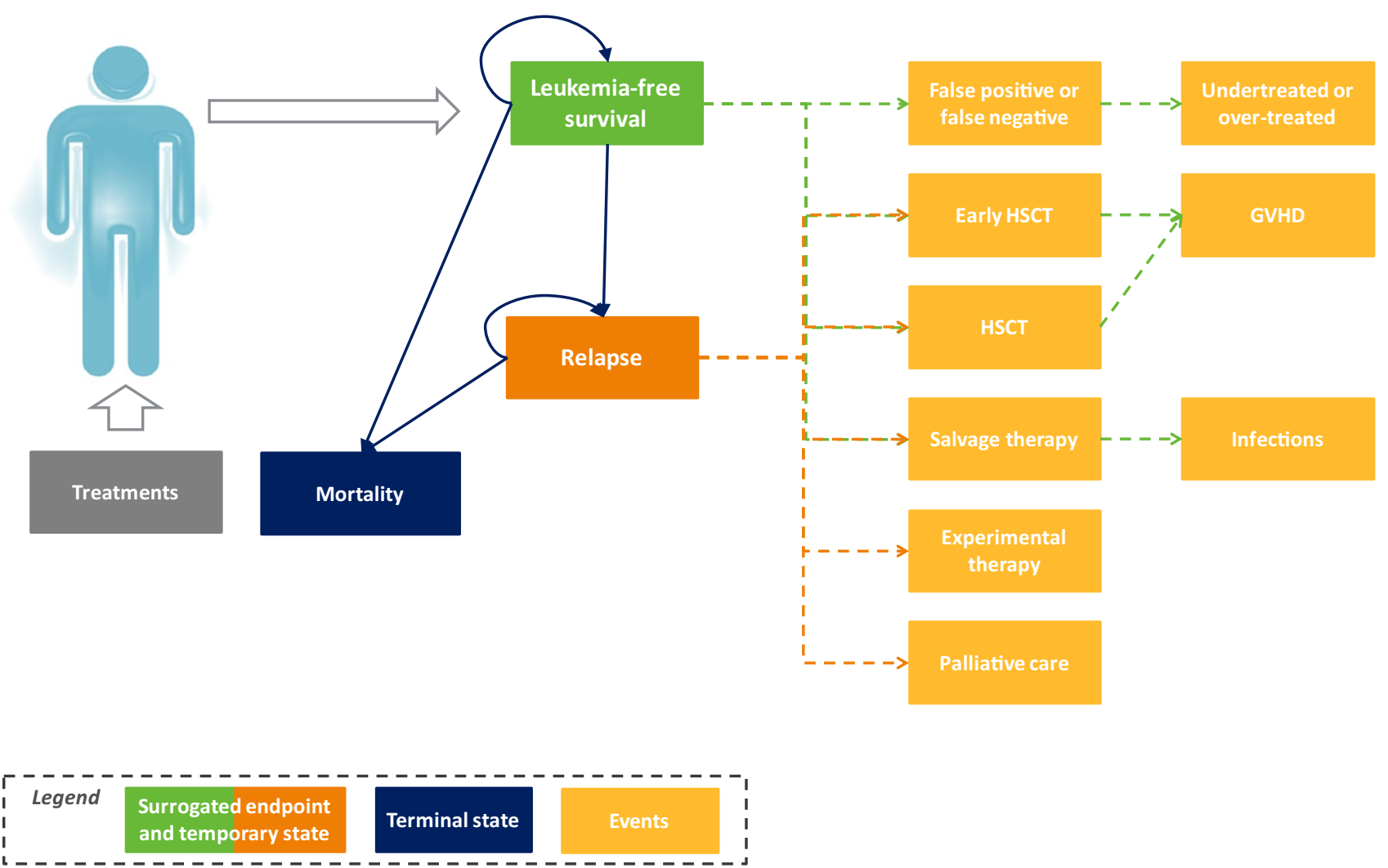

Fig. 1 Model structure. GVHD graft vs. host disease, HSCT hematopoietic stem cell transplantation 
chemotherapy, subsequent relapses, potential HSCT, graft vs. host disease [GVHD], and palliative care). The model was based on patient-level data obtained from the Quebec Leukemia Cell Bank database, which included specific baseline characteristics such as age and cytogenetic risk group, a hypothetical treatment pathway resulting from the introduction of the new HMGA2 prognostic test (Fig. 2) and patient outcomes [13]. Variables used in the model included age ( $<60$ and $\geq 60$ years old), cytogenetic risk group (favorable, intermediate, and adverse), HMGA2 expression profile (HMGA2 positive or negative), early HSCT (in first $\mathrm{CR}$ ), relapse, and late HSCT (HSCT in second CR or after relapse). As these factors were included as variables in the model, the impact of age, cytogenetic risk group, HMGA2 expression, and HSCT (early HSCT or late HSCT) on OS and LFS was therefore included in the model in addition to the associated costs. Therefore, the post-relapse state and post-HSCT states are incorporated in the efficacy results.

The model was developed for a public healthcare setting in Canada in 2019. A publicly funded healthcare system perspective was employed as per the Canadian Agency for Drugs and Technologies in Health guidelines for economic evaluations and a societal perspective as an additional scenario. A lifetime horizon was modeled and a $1.5 \%$ discount rate was applied to cost and QALYs as per Canadian Agency for Drugs and Technologies in Health guidelines [17].

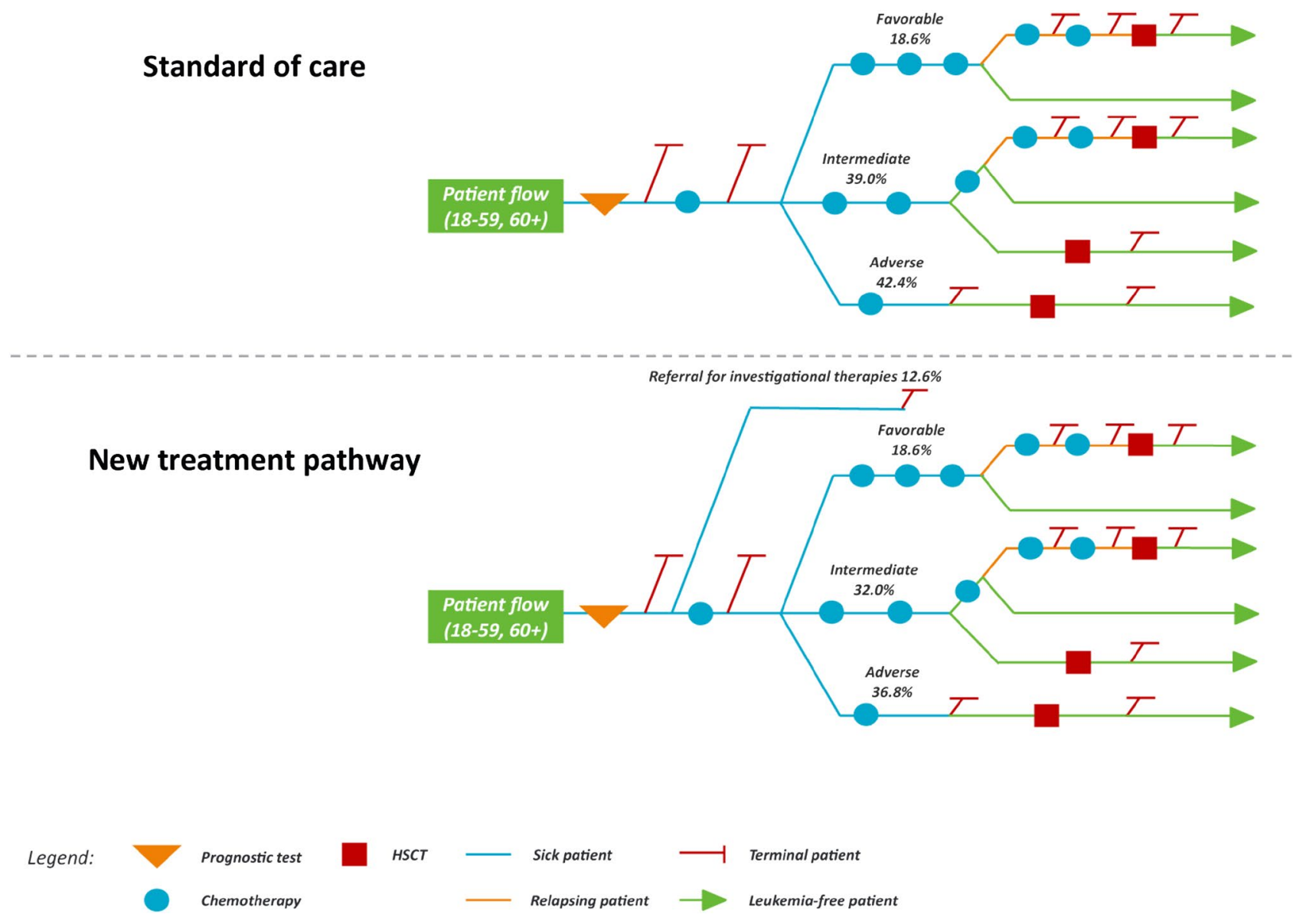

Fig. 2 Current treatment pathway with the standard of care and hypothetical new treatment pathway following the introduction of the HMGA2 prognostic test. Patients with acute myeloid leukemia eligible for intensive chemotherapy (blue circle) receive one or two cycles of induction chemotherapy after leukemia diagnosis and two to four cycles of consolidation chemotherapy after achieving complete remission. Patients who are candidates for hematopoietic stem cell transplantation (HSCT) in first remission (intermediate- and adverse-risk patients) can receive one to two cycles of consolidation chemotherapy before transplantation if a suitable donor is not readily available [10].
The HMGA2 test used in the new treatment pathway reclassified $17 \%$ of intermediate-risk patients $(\sim 7 \%$ of all patients with acute myeloid leukemia) into the adverse-risk category. Accordingly, 39\% of patients are classified in the intermediate-risk category in the standard of care pathway compared with $32 \%$ in the new treatment pathway. Moreover, $\sim 12.6 \%$ of all patients with acute myeloid leukemia in the new treatment pathway (adverse-risk patients with a positive HMGA2 test) are unresponsive to standard intensive therapies and can be referred earlier for investigational therapies 


\subsection{Model Assumptions}

The model structure was based on a treatment pathway developed and validated through consultation with five AML clinicians and HSCT experts to ensure an accurate reflection of clinical practice in Canada. This process resulted in the inclusion of potential decision branches not often represented in models, based on the following assumptions:

- Patients dying before the first month of chemotherapy (first month mortality data used as a proxy).

- Patients dying after first month of chemotherapy, but before any potential changes can be made to the treatment pathway (second month mortality data used as a proxy).

- Prognostic-based decision not to treat with standard intensive therapy; supported by the additional information on the patient potential outcomes, i.e., patient-level data on patients with adverse factors such as adverse genetic risk group and high expression of $H M G A 2$.

- The model does not differentiate the transition rate by treatment, but only by health state. Therefore, the difference in efficacy between arms results from the time spent (area under the curve) in different health states resulting from relapse, HSCT, or other event rates. Consequently, the test itself does not directly improve OS or LFS but leads to informed decision making for early HSCT or a change in the treatment pathway that will result in improved efficacy or modified costs.

The probability of having an event, i.e., progression/ relapse, OS, and HSCT, was determined only by the risk factor and the age groups of patients (as the use of the HMGA2 test does not influence the efficacy of treatments) and was based on Quebec Leukemia Cell Bank patient-level data [13], the Leucegene meta-analysis, and literature sources (see Table S1 of the ESM). Under the new hypothetical treatment pathway (Fig. 2), based on their gene signature (i.e., $H M G A 2$ expression), a subgroup of patients will be referred to receive investigational therapies. These patients, representing $12.6 \%$ of patients with AML in Marquis et al. [13], were resistant to AML standard intensive therapy and no patients in this subgroup were long-term survivors. These patients could therefore be assigned to investigational therapies in clinical trials early after genetic testing, as the currently available treatments would have no positive clinical outcome, but would still have a number of adverse events. Additionally, a subgroup of patients in the intermediate-risk group will be re-stratified into the adverse-risk group. As a result, the probabilities presented in Table S1 of the ESM are not dependent on the treatment arm and thus, apply to patients treated with the standard approach or with a strategy using the HMGA2 prognostic test.

\subsection{Outcomes}

The model included expected final and surrogated endpoints over a lifetime horizon such as LFS (in months and years), OS (in months and years), quality-adjusted months, and QALYs. The main clinical endpoints used in the model (LFS and OS) were based on patient-level data and were used to generate additional data on the disease status and health state of patients. While the model was based on three mutually exclusive states, additional events were added to estimate additional resource use and costs. The events were all modeled as one-time events with a duration based on key opinion leader (KOL) assumptions applied to both the cost and utility decrement. For false-negative and false-positive patients revealed through subsequent routine testing, a re-evaluation of the patient status and diagnosis is needed to assign a new treatment pathway. Therefore, the patients must go through testing again and an event disutility was applied. Effectiveness measures were calculated on a discounted and undiscounted basis from a public healthcare system or societal perspective and included cost per QALY and cost per LY.

\subsection{Costs}

All costs were expressed as 2019 Canadian dollars and adjusted for inflation using the Canadian healthcare consumer price index as necessary [18]. Any costs expressed in other currencies than Canadian dollars were converted using a purchasing power parity index for the year of the cost and adjusted for inflation as necessary.

\subsubsection{Healthcare Resource Utilization and Costs}

The diagnostic testing costs for the standard of care (presented in Table S8 of the ESM, diagnostic costs category) were obtained from a published study by Uyl-de Groot et al. and adjusted for inflation to 2019 Canadian dollars as described [19]. The cost of the HMGA2 prognostic test was based on Leucegene internal price sources assumptions for 2019. The HMGA2 laboratory test cost (from KOL opinion) is generated based on the material needed in the laboratory, interpretation, and communication of the results. The HMGA2 test result will be communicated during a visit with the hematologist, which is included in the follow-up and routine care costs.

Chemotherapy drug costs were estimated in CAD per milligram using commercially available vial dosages and prices available from the Ontario Drug Benefit formulary, the Quebec Health Insurance (Régie de l'assurance maladie du Québec) database, and Selleck Chemicals (drug manufacturer; see Table S2 of the ESM). Vial dosages were obtained from Health Canada monographs or Selleck Chemicals. Chemotherapy utilization was based on a physician survey and clinician validation (see Table $\mathrm{S} 3$ of the ESM). This was performed using a 
simple online survey to collect five clinician perspectives on drug treatment currently used in clinical practice. The data captured for healthcare utilization and AML treatments are presented in Tables S4 and S5 of the ESM, respectively.

Healthcare resource utilization costs were calculated per health state to allow the inclusion of routine care for patients with AML based on their health state (see Table S6 of the ESM). The healthcare resource utilization was based on Reed et al. [20], identifying chronic and accelerated phases, which were respectively used to correspond to the prerelapse state and the post-relapse state in the model. Costs associated with HSCT were obtained from the Ontario Case Costing Initiative (OCCI) for the year 2016/2017 using the CMG Grouper 610. The costs included in the model were $\$ 59,033$ CAD per HSCT after inflation [21]. Other events costs were also obtained from the OCCI database, such as GVHD, relapse, and infection.

\subsubsection{Indirect Costs}

The indirect costs included in the model were calculated using a friction cost approach and were based on the annual average salary in Canada in 2019 ( $\$ 55,889$ CAD). In a friction approach, premature deaths cause a "friction" period during which the employer must find and train a replacement for the deceased employee. The duration of friction was 6 months and included a reduction in activity based on baseline participation rates for persons under 59 years of age $(0.777)$ and over 60 years of age (0.357), in addition to a $1.96 \%$ reduction in the activity rate per year based on the same data. Therefore, for any premature death, a friction cost equivalent of 6 months' salary was applied for the employed population.

\subsection{Utilities}

A targeted literature search was performed to identify the utility values available in AML. The search targeted the utility value per health state in AML (pre-progression with 0.66 , and post-progression with 0.53 ) in addition to event disutility, such as GVHD $(-0.26)$, relapse $(-0.05)$, infection (- 0.31), early HSCT $(0.08)$, late HSCT $(0.08)$, false negative $(-0.02)$, false positive $(-0.01)$, and on treatment (0.09). Health utility values for different events were obtained from published literature sources, and event duration was based on consultation with five AML clinicians and HSCT experts (see Table S7 of the ESM).

\subsection{Sensitivity Analyses}

\subsubsection{One-Way Sensitivity Analyses}

One-way sensitivity analyses were conducted to explore the effect of uncertainty in the model parameters on the incremental cost-effectiveness ratio (ICER) by changing the values of key parameters. Discount rates, medical and treatment costs, utility values, efficacy parameters, and event rates were varied between the $95 \%$ confidence intervals using a range based on the standard error with the base-case values as the center of the distribution. In order of preference, the standard error was based on the reference when available, estimated using the standard deviation, or estimated based on the maximum/minimum range of the value, or assumed to be similar to the distribution in the most related OCCI cost (for costs only). Furthermore, the prognostic test cost was both doubled and reduced by half in an attempt to determine the impact of a range of different test costs.

Several scenarios tested the impact of varying the utility levels on the overall results, and were all evaluated using the $95 \%$ confidence intervals (1.96 times the standard error in both directions): all events utility, no 'on treatment' utility, LFS utility, post-relapse utility, and all utility. Similarly, sensitivity analyses were conducted on the disutility values associated with events rate for false negative, false positive, experimental therapy, infections, relapse, early or late HSCT, and GVHD.

\subsubsection{Probabilistic Sensitivity Analyses}

Probabilistic sensitivity analyses (PSA) were conducted to assess the cost effectiveness of the HMGA2 prognostic test where the uncertainty surrounding multiple input variables was examined simultaneously. Therefore, this step allowed the construction of the cost-effectiveness plane and the costeffectiveness acceptability curve. The value of each input variable examined in the PSA was varied by their standard error, which was determined in the same manner as for the DSA. Variability estimates for costs were estimated based on relevant OCCI cost categories (e.g., unit costs), but available from the original sources for important effectiveness variables (OS, LFS, relapse, HSCT, GVHD, utility). The base-case values and the distributions used to vary each variable are shown in Table 1. Each scenario analysis employed 10,000 iterations.

\subsection{Model Validation}

The model was validated using KOL interviews and a KOL/ clinician survey. The model was reviewed by a quality-control agent for internal validity. In addition, the protocol and the model were reviewed by an advisory committee composed of clinicians and economists. The external validity of the effectiveness estimations was confirmed using a metaanalysis of 50 clinical trials to determine if the real-world evidence generated similar results to the literature (Unpublished Leucegene report) [23]. The external validity was 
tested using a targeted literature of diagnostic test economic evaluations, more specifically in AML.

\section{Results}

\subsection{Study Parameters and Total Costs}

According to the model results, implementation of the HMGA2 prognostic test was projected to lead to increases in both LFS and OS over the lifetime of a patients with AML (Table 2). The expected differences over a lifetime horizon between the standard of care and the HMGA2 prognostic test in LFS and OS were 1.92 and 3.12 months, respectively, in favor of the HMGA2 prognostic test.

Table S8 of the ESM presents the estimated healthcare utilization costs associated with the standard of care and the HMGA2 prognostic test at 2, 5, and 10 years and throughout the lifetime of a patient with AML. Expected total lifetime societal costs were \$161,358 CAD with the current standard of care and \$151,908 CAD with the new test. In general, the costs associated with the standard of care were higher than the costs associated with the HMGA2 prognostic test irrespective of the time horizon. The only exceptions included diagnostic costs, routine medical costs, and costs as a result of infections and false positives (see Table S8 of the ESM).

\subsection{Base-Case Results}

Under the base-case assumptions (see Table S9 of the ESM), the HMGA2 prognostic test was estimated to lead to increases in QALYs (discounted) of 0.138 over a lifetime for a patient with AML. The incremental lifetime cost difference per patient between the HMGA2 prognostic test and the standard of care was \$9270 CAD from the public healthcare perspective, and \$9451 CAD when including a societal perspective (see Table S10 of the ESM). The incremental LYs and QALYs with the HMGA2 prognostic test from public healthcare and societal perspectives were 0.260 and 0.138 , respectively, which led to dominance over the standard of care. This dominance resulted in negative ICERs, which were estimated respectively for the payer and societal perspectives at $-\$ 35,689 \mathrm{CAD}$ and $-\$ 36,384 \mathrm{CAD}$ for the cost per LY and at - \$67,138 CAD and - \$68,445 CAD for the cost per QALY (Table S10 of the ESM). The ICERs presented above indicate dominance, but must be interpreted with caution.

\subsection{Sensitivity Analyses}

The deterministic sensitivity analyses conducted show the new treatment strategy to be dominant and demonstrate the robustness of the base-case results (Table 3). The most influential scenarios included the addition of hazard ratios (HRs), in particular for OS, which affected the partition data by changing the effect modifiers (Fig. 3).

The cost-effectiveness plane and cost-effectiveness acceptability curve of the PSA are presented in Fig. 4 and Fig. S2 of the ESM, respectively. The majority of simulations are clustered on the southern quadrants of the costeffectiveness plane, predominantly on the eastern side (Fig. 4). The cost-effectiveness acceptability curve shows that for willingness-to-pay thresholds of \$50,000 CAD and $\$ 100,000$ CAD, the probability of the HMGA2 prognostic test being cost effective is $79.18 \%$ and $87.19 \%$, respectively (see Fig. S2 of the ESM).

\section{Discussion}

In this analysis, we have demonstrated the cost effectiveness of a new AML prognostic test compared with the current standard of care in Canada. This validated HMGA2 test is performed using a technology already available in most molecular diagnostic laboratories and is rapid and inexpensive.

This test will allow AML physicians to re-stratify a subgroup of intermediate-risk patients into the adverserisk group and to refer another group of patients to receive investigational therapies as a result of its ability to predict resistance to standard intensive therapies. The analysis was conducted from a healthcare payer and societal perspective, and shows the new test to be a dominant strategy, with a cost per QALY of $-\$ 67,138$ CAD and $-\$ 68,445$ CAD, respectively.

The HMGA2 prognostic test allows for re-stratification of approximately $17 \%$ of intermediate-risk patients. These patients are reclassified into the adverse-risk group, which leads to a decrease in unnecessary treatments and possibly, to earlier referral of patients for allogeneic HSCT in first remission. The reduction in administered treatments not only results in important costs savings and thus, influences the cost per QALY, but also reduces the disutility associated with re-induction chemotherapy if the patient relapses, and optimizes the sequence of effective treatments. Additionally, re-stratification of patients has a positive influence on the ICERs by reducing the costs associated with treatments such as HSCT (including early HSCT) and improving quality of life through better HSCT response and success as well as avoidance of infections and other adverse events.

As with any health economic model, our analysis has several limitations that need to be addressed. First, our study did not include clinical trial data, but rather it was based on patient-level data from the Quebec Leukemia Cell Bank, a biobank certified by the Canadian Tissue Repository Network. While the absence of a clinical trial can be 
Table 1 Input variables assessed in probabilistic sensitivity analyses

\begin{tabular}{|c|c|c|c|c|}
\hline Variable & Base-case value & SE & Distribution & Distribution source \\
\hline \multicolumn{5}{|l|}{ Costs } \\
\hline HMGA2 prognostic test (CAD) & 100 & $10 \%$ & Normal & Assumption \\
\hline All drug costs (CAD) & $\begin{array}{l}\text { Induction }=1777 \\
\text { Consolidation } 1=3912 \\
\text { Relapse }=1517 \\
\text { Consolidation } 2=1655\end{array}$ & $8 \%$ & Gamma & $\begin{array}{l}\text { SD from OCCI AML treatments: principal procedure } \\
\text { 1ZZ35HAM1 for patients with AML }\end{array}$ \\
\hline Pre-relapse medical costs (CAD) & 234 & $4 \%$ & Gamma & $\begin{array}{l}\text { SD from OCCI AML treatments: principal procedure } \\
\text { 1ZZ35HAM1 for patients with AML }\end{array}$ \\
\hline Post-relapse medical costs (CAD) & 853 & $4 \%$ & Gamma & $\begin{array}{l}\text { SD from OCCI AML treatments: principal procedure } \\
\text { 1ZZ35HAM1 for patients with AML }\end{array}$ \\
\hline \multicolumn{5}{|l|}{ Utility } \\
\hline LFS utility & 0.66 & 0.02 & Beta & $\begin{array}{l}\text { Assumption: the min and max represent the CIs }( \pm 1.96 * \\
\text { SD), Leunis } 2015 \text { [22] }\end{array}$ \\
\hline Post-relapse utility & 0.53 & 0.017 & Beta & $\begin{array}{l}\text { Assumption: the min and max represent the CIs }( \pm 1.96 * \\
\text { SD), Leunis } 2015 \text { [22] }\end{array}$ \\
\hline \multicolumn{5}{|l|}{ Standard of care efficacy } \\
\hline OS HR ${ }^{\mathrm{a}}$ (months) & 4.5 & 0.25 & Log-normal & SE from Leucegene patient-level data \\
\hline LFS HR ${ }^{\mathrm{a}}$ (months) & 4.67 & 0.23 & Log-normal & SE from Leucegene patient-level data \\
\hline \multicolumn{5}{|l|}{ New prognostic test efficacy } \\
\hline $\mathrm{OS} \mathrm{HR}^{\mathrm{a}}$ (months) & 4.1 & 0.25 & Log-normal & SE from Leucegene patient-level data \\
\hline LFS HR ${ }^{\mathrm{a}}$ (months) & 4.47 & 0.23 & Log-normal & SE from Leucegene patient-level data \\
\hline \multicolumn{5}{|l|}{ Standard of care events rate } \\
\hline Relapse rate & $55.72 \%$ & $25 \%$ & Log-normal & Leucegene patient-level data \\
\hline Early HSCT rate & $19.54 \%$ & $16 \%$ & Log-normal & Leucegene patient-level data \\
\hline Late $\mathrm{HSCT}^{\mathrm{b}}$ rate & $23.86 \%$ & $18 \%$ & Log-normal & Leucegene patient-level data \\
\hline GVHD rate & $57.86 \%$ & $24 \%$ & Log-normal & Leucegene patient-level data \\
\hline \multicolumn{5}{|l|}{ New prognostic test events rate } \\
\hline Relapse rate & $49.35 \%$ & $25 \%$ & Log-normal & Leucegene patient-level data \\
\hline Early HSCT rate & $12.41 \%$ & $16 \%$ & Log-normal & Leucegene patient-level data \\
\hline Late $\mathrm{HSCT}^{\mathrm{b}}$ rate & $12.98 \%$ & $18 \%$ & Log-normal & Leucegene patient-level data \\
\hline GVHD rate & $50.78 \%$ & $24 \%$ & Log-normal & Leucegene patient-level data \\
\hline
\end{tabular}

$A M L$ acute myeloid leukemia, $C A D$ Canadian dollars, $C I s$ confidence intervals, GVHD graft versus host disease, $H R$ hazard ratio, $H S C T$ hematopoietic stem cell transplantation, $L F S$ leukemia-free survival, max maximum, min minimum, $O S$ overall survival, $S D$ standard deviation, $S E$ standard error

${ }^{\text {a}}$ Final HR compares to patients in the most favorable risk, age, HSCT, relapse, and genomic group (all at one time)

${ }^{\mathrm{b}}$ Defined as HSCT in second complete remission or after relapse

Table 2 Total quality-adjusted life-years (QALYs) and lifeyears (LYs): discounted

\begin{tabular}{|c|c|c|c|c|c|c|c|c|}
\hline & \multicolumn{4}{|c|}{ Standard of care } & \multicolumn{4}{|c|}{ New prognostic test } \\
\hline & 2 years & 5 years & 10 years & Lifetime & 2 years & 5 years & 10 years & Lifetime \\
\hline LFS (months) & 16.23 & 26.46 & 32.24 & 35.17 & 16.48 & 27.25 & 33.61 & 37.09 \\
\hline LFS (LYs) & 1.35 & 2.20 & 2.69 & 2.93 & 1.37 & 2.27 & 2.80 & 3.09 \\
\hline OS (months) & 18.10 & 32.37 & 42.61 & 50.20 & 18.34 & 33.25 & 44.39 & 53.32 \\
\hline OS (LYs) & 1.51 & 2.70 & 3.55 & 4.18 & 1.53 & 2.77 & 3.70 & 4.44 \\
\hline QALYs & 1.03 & 1.78 & 2.30 & 2.66 & 1.03 & 1.81 & 2.37 & 2.80 \\
\hline
\end{tabular}

$L F S$ leukemia-free survival, $L Y$ life-year, $O S$ overall survival, $Q A L Y$ quality-adjusted life-year considered a major limitation, the patient-level data from the Quebec Leukemia Cell Bank database have been compiled using adult patient medical records from nine university and regional hospitals. The absence of clinical trials also increases the uncertainty in the PSA results. Furthermore, as these data were collected from real-world settings, we 
Table 3 Deterministic sensitivity analyses scenarios and incremental costeffectiveness ratios [ICERs, (Canadian dollars, CAD)] generated from conservative and optimistic parameter ranging

\begin{tabular}{|c|c|c|c|c|}
\hline Scenarios & Conservative ICER & Base-case ICER & Optimistic ICER & Difference \\
\hline OS HR & $-27,409$ & $-67,138$ & $-157,386$ & 129,976 \\
\hline Risk group HR & $-47,348$ & $-67,138$ & $-89,751$ & 42,403 \\
\hline HMGA2 prognostic test cost & $-41,098$ & $-67,138$ & $-69,742$ & 28,644 \\
\hline All discounting rates & $-53,874$ & $-67,138$ & $-81,173$ & 27,299 \\
\hline Benefits discounting rate & $-55,554$ & $-67,138$ & $-79,806$ & 24,252 \\
\hline PFS HR & $-72,207$ & $-67,138$ & $-56,252$ & 15,955 \\
\hline All drug costs & $-59,986$ & $-67,138$ & $-74,289$ & 14,303 \\
\hline Genomic group HR & $-60,858$ & $-67,138$ & $-74,238$ & 13,380 \\
\hline No "on treatment" utility & $-58,507$ & $-67,138$ & $-67,749$ & 9242 \\
\hline All utility & $-71,527$ & $-67,138$ & $-63,256$ & 8272 \\
\hline Late HSCT rate & $-63,400$ & $-67,138$ & $-70,875$ & 7475 \\
\hline Age group HR & $-63,606$ & $-67,138$ & $-70,744$ & 7138 \\
\hline Relapse HR & $-64,016$ & $-67,138$ & $-70,392$ & 6376 \\
\hline LFS utility & $-70,357$ & $-67,138$ & $-64,200$ & 6157 \\
\hline HSCT costs & $-64,306$ & $-67,138$ & $-69,969$ & 5663 \\
\hline Early HSCT rate & $-64,516$ & $-67,138$ & $-69,760$ & 5244 \\
\hline Relapse rate & $-64,862$ & $-67,138$ & $-69,477$ & 4614 \\
\hline Progressive utility & $-68,789$ & $-67,138$ & $-65,564$ & 3225 \\
\hline Cost discounting rate & $-65,108$ & $-67,138$ & $-68,288$ & 3180 \\
\hline Relapse + drug costs & $-65,827$ & $-67,138$ & $-68,449$ & 2622 \\
\hline Induction drug costs & $-65,993$ & $-67,138$ & $-68,283$ & 2290 \\
\hline Post-progression medical costs & $-67,740$ & $-67,138$ & $-66,536$ & 1204 \\
\hline False positive rate & $-67,674$ & $-67,138$ & $-66,602$ & 1072 \\
\hline Pre-progression medical costs & $-67,401$ & $-67,138$ & $-66,875$ & 526 \\
\hline Palliative care costs & $-66,890$ & $-67,138$ & $-67,385$ & 495 \\
\hline GVHD costs & $-67,144$ & $-67,138$ & $-67,132$ & 11 \\
\hline GVHD rate & $-67,143$ & $-67,138$ & $-67,132$ & 11 \\
\hline Relapse costs & $-67,137$ & $-67,138$ & $-67,139$ & 2 \\
\hline False negative rate & $-67,138$ & $-67,138$ & $-67,137$ & 1 \\
\hline Infections rate & $-67,138$ & $-67,138$ & $-67,138$ & 0 \\
\hline All events disutility & $-67,138$ & $-67,138$ & $-67,138$ & 0 \\
\hline Infection costs & $-67,138$ & $-67,138$ & $-67,138$ & 0 \\
\hline Experimental therapy rate & $-67,138$ & $-67,138$ & $-67,138$ & 0 \\
\hline
\end{tabular}

$C A D$ Canadian dollars, GVHD graft versus host disease, HR hazard ratio, HSCT hematopoietic stem cell transplantation, ICER incremental cost-effectiveness ratio, LFS leukemia-free survival, $N P T$ new prognostic test, $O S$ overall survival, $P F S$ progression-free survival believe our analysis and its outputs reflect real-world clinical practice and outcomes. A patient-level model can capture the history of individual patients, which is very important in personalized medicine. The retrieval of real evidence allows for data capture regarding effectiveness rather than efficacy. This is of high importance as it is often only possible to obtain efficacy data from randomized clinical trials. In these cases, the efficacy data are collected from "ideal settings" with highly compliant patients and does not reflect the realworld settings under normal clinical conditions.

Another limitation of our analysis was the lack of qualityof-life data collection and our reliance on obtaining this type of data from various literature sources. The available utility values were compared and selected based on the quality of the data and time since publication to reflect the most up-to-date health states. Furthermore, utility values based on assumptions were avoided as much as possible to prevent creating any biases. Our analysis included the modeling of the complete treatment pathway, rather than just a subset or branch as is the case with most economic analyses. This is likely to have introduced further uncertainty as patients were modeled through a lifetime horizon and multiple stages of the treatment pathway.

However, we have tried to minimize uncertainty as much as possible by ensuring that the treatment pathway modeled in our analysis and the resource use data inputted were based on clinician validation. Similarly, the clinical data inputted 


\begin{tabular}{|c|c|c|}
\hline OS HR $\quad-157,386$ & 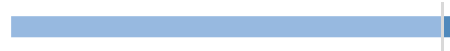 & 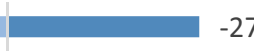 \\
\hline Risk group HR & $-89,751$ & $-47,348$ \\
\hline HMGA2 Diagnostic test cost & $-69,742$ & $-41,098$ \\
\hline All discounting rates & $-81,173$ & $-53,874$ \\
\hline Benefits discounting rate & $-79,806$ & $-55,554$ \\
\hline PFS HR & $-72,207$ & $-56,252$ \\
\hline All Drug costs & $-74,289$ & $-59,986$ \\
\hline Genomic group HR & $-74,238$ & $-60,858$ \\
\hline No "on treatment" utility & $-67,749$ & $-58,507$ \\
\hline All utility & $-71,527$ & $-63,256$ \\
\hline Late HSCT rate & $-70,875$ & $-63,400$ \\
\hline Age group HR & $-70,744$ & $-63,606$ \\
\hline Relapse HR & $-70,392$ & $-64,016$ \\
\hline LFS utility & $-70,357$ & $-64,200$ \\
\hline HSCT costs & $-69,969$ & $-64,306$ \\
\hline Early HSCT rate & $-69,760$ & $-64,516$ \\
\hline Relapse rate & $-69,477$ & $-64,862$ \\
\hline Progressive utility & $-68,789$ & II $-65,564$ \\
\hline Cost discounting rate & $-68,288$ & $-65,108$ \\
\hline Relapse+ drug costs & $-68,449$ & || $-65,827$ \\
\hline Induction drug costs & $-68,283$ & || $-65,993$ \\
\hline Post-progression medical costs & $-67,740$ & $\|-66,536$ \\
\hline False postitive rate & $-67,674$ & $\|-66,602$ \\
\hline Pre-progression medical costs & $-67,401$ & $-66,875$ \\
\hline Palliative care costs & $-67,385$ & $-66,890$ \\
\hline GVHD costs & $-67,132$ & $-67,144$ \\
\hline GVHD rate & $-67,132$ & $-67,143$ \\
\hline Relapse costs & $-67,137$ & $-67,139$ \\
\hline False negative rate & $-67,137$ & $-67,138$ \\
\hline Infections rate & & $-67,138$ \\
\hline All events disutility & & $-67,138$ \\
\hline Infection costs & & $-67,138$ \\
\hline Experimental therapy rate & & $-67,138$ \\
\hline$-190,000$ & $\begin{array}{c}-90,000 \\
\text { ICER (Cost-per-Q }\end{array}$ & $\begin{array}{r}-40,000 \\
Q A L Y) \text { in CAD\$ }\end{array}$ \\
\hline
\end{tabular}

Note: Light and dark blue bars respectively represent the upper and lower bound estimates of the $95 \%$ Confidence interval for each scenario

Fig. 3 Incremental cost-effectiveness ratio tornado diagram for multiple deterministic sensitivity analyses (Canadian dollars [CAD]). $G V H D$ graft vs. host disease, $H R$ hazard ratio, $H S C T$ hematopoietic stem cell transplantation, ICER incremental cost-effectiveness ratio,

in the model were externally validated through comparisons with a meta-analysis of more than 50 studies (unpublished Leucegene report) [23]. Furthermore, both deterministic sensitivity analyses and PSAs were conducted to test the
LFS leukemia-free survival, $O S$ overall survival, $P F S$ progressionfree survival, $Q A L Y s$ quality-adjusted life-years. Late HSCT was defined as HSCT in second complete remission or after relapse

stability of the base-case results and check for potential variables that might influence the base-case scenario. By modeling patients through the complete treatment pathway from diagnosis to end-of-life care and including all phases 


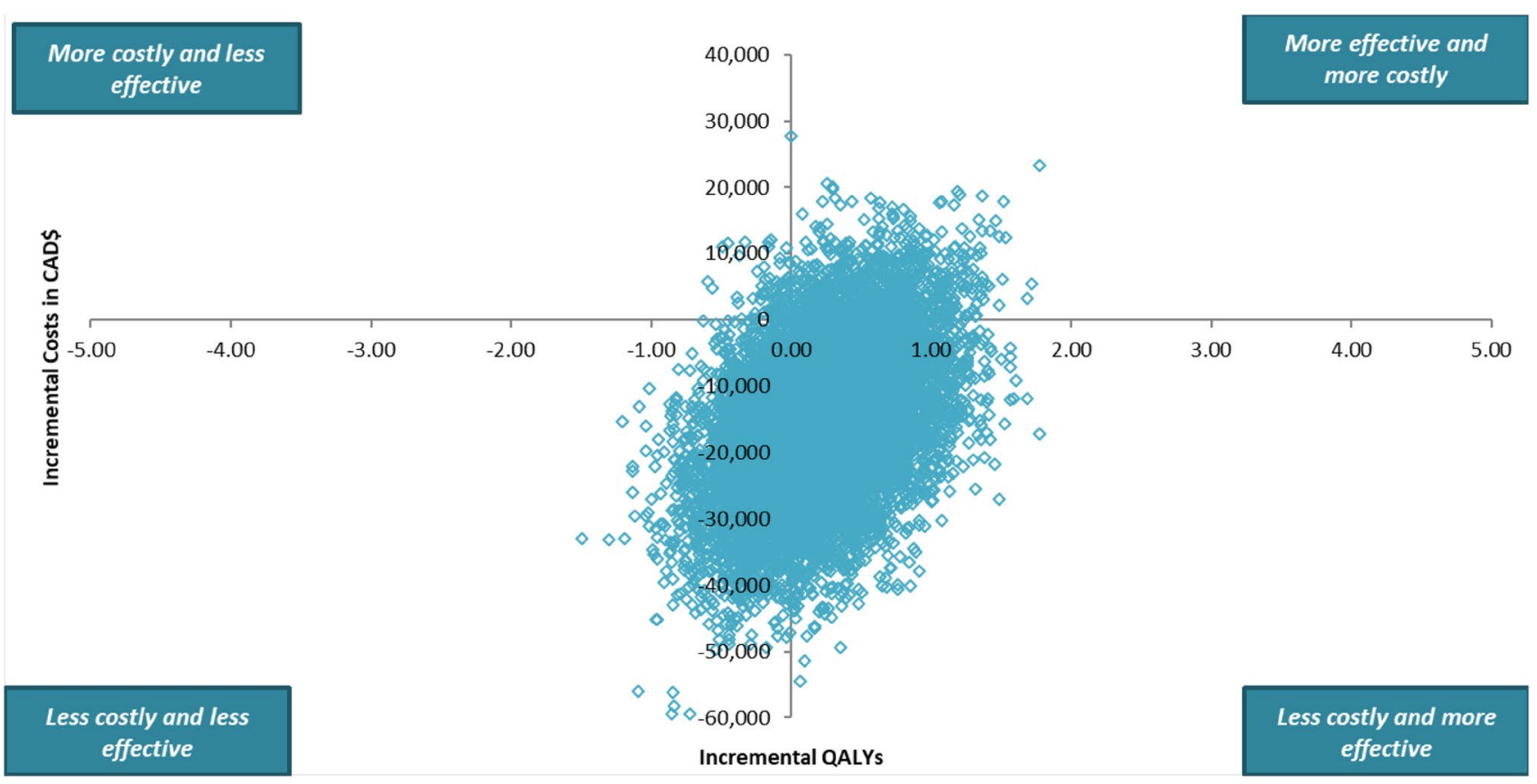

Fig. 4 Cost-effectiveness plane of the probabilistic sensitivity analysis for comparison between the HMGA2 prognostic test and the standard of care. CAD Canadian dollars, $Q A L Y s$ quality-adjusted life-years

of chemotherapy and leukemia-related events, our study presents the potential impact of the HMGA2 prognostic test on physician choice and lifetime outcomes on patients with AML and healthcare systems. Last, in the absence of longterm data, the patient-level data relating to OS and LFS were extrapolated to cover the lifetime horizon of a patient with AML and as a result, the effects of the HMGA2 prognostic test were based on assumptions.

Our cost-effectiveness analysis may also have a number of limitations related to the generalizability of the results. First, while we included indirect costs in our analysis, they are mainly related to productivity costs and do not include caregiver costs, travel costs, or other out-of-pocket costs incurred by the patient. It is unlikely that the inclusion of such costs would dramatically affect the overall results of the analysis; however, given the improvements in LFS and OS estimated for the HMGA2 prognostic test they would likely be incurred at a similar rate while patients are alive and thus, result in a decrease in dominance. Second, our use of patient-level data from the Quebec Leukemia Cell Bank greatly limits the generalizability of the results to other patient populations than Canadian or North American patients. Third, some of the unit costs were only available for the Quebec province while others were only available for Ontario, which could have slightly different local unit costs. Finally, the comparator used in the study was the standard of care, and the currently available data in the literature are not sufficient to compare to other potential tests separately from the standard of care where some tests are currently used.
To our knowledge, the only previous analysis describing the impact of genomic diagnostics in a Canadian setting is a cost-effectiveness decision analytic model developed by Cressman et al. [24]. The results of their analysis are somewhat comparable to ours as they also assessed the cost effectiveness of stratifying patients with intermediate-risk AML over a 10-year time horizon. They estimated that genomic analysis would result in increased use of first-remission allogeneic stem cell transplantation leading to an additional cost of $\$ 12,556$ (2013 CAD) and 0.26 QALYs over the standard of care, while our analysis estimated an incremental cost of - \$10,687 (2019 CAD) and 0.073 QALYs (discounted) during a 10-year time horizon (owing to re-stratification of patients to investigational therapies and earlier HSCT in first remission) [24]. However, the difference may be attributed to the use of a multigene stratification system of ten selected prognostic genes, which may be more accurate in reclassifying intermediate-risk group patients than the single-gene system $\left(H M G A 2^{-} / H M G A 2^{+}\right)$assessed in our model. Despite the potential accuracy difference, PSAs showed the HMGA2 prognostic test described in our analysis had a higher probability of being cost effective (87.19\%) at a willingness-to-pay threshold of $\$ 100,000$ CAD compared with the multigene system (58\%) [24].

It is important to note that the uncertainty in the PSA results is relatively high, which can be attributed to uncertainty in the effectiveness of the treatment pathway. The effectiveness uncertainty also resulted in the spreading of the simulation across the four quadrants (Fig. 4), with 
around $8.17 \%$ of the simulations in quadrant I (incremental cost effectiveness), $0.8 \%$ of the simulations in quadrant II (dominated), $27.39 \%$ of the simulations in quadrant III (decremental cost effectiveness), and $63.64 \%$ of the simulations in quadrant IV (dominant).

\section{Conclusions}

It is important that healthcare providers offer the most suitable therapies for AML that can treat the disease effectively and minimize the risk of relapse. Appropriate stratification of patients to treatments can not only result in improved outcomes, but may also be a cost-effective means of healthcare resource utilization. Our analysis has demonstrated the cost effectiveness of a HMGA2 prognostic test used to stratify patients with AML to treatment by genetic risk group in Canada, but show some uncertainty when analyzed in the PSA. When compared to the standard of care, the new test was estimated to improve both LFS and OS, and result in lower overall costs, from a healthcare system and societal perspective. These findings provide important information for decision makers when allocating funding for the HMGA2 prognostic test for patients with AML.

Acknowledgements The authors acknowledge all patients and hematologists who participated to the Quebec Leukemia Cell Bank (Banque de cellules leucémiques du Québec, BCLQ) and the BCLQ team who characterized and provided AML samples of the Leucegene cohort. We acknowledge Vincent-Philippe Lavallée for his contribution to the identification of the HMGA2 marker, Sébastien Lemieux for supervision of the bioinformatics team for the Leucegene project, Geneviève Boucher and Patrick Gendron for RNA sequencing data processing (IRIC bioinformatics platform), and Muriel Draoui for the Leucegene project coordination. The authors also acknowledge George Braileanu (Adelphi Values Limited) and Jaclyn Hearnden (Purple Squirrel Economics) for medical writing assistance in drafting the manuscript.

Author contributions GT developed the cost-effectiveness model used for this analysis and contributed to the development of this manuscript. BR contributed to the review of the model and to the development of this manuscript. MM developed and validated the HMGA2 test, performed tests and analyses for the Leucegene patient cohort, and edited this manuscript. CB performed statistical analyses for the Leucegene patient cohort. GS contributed to project conception and identification of the HMGA2 marker. JH contributed to project conception and identification of the HMGA2 marker, the cost-effectiveness model used for this analysis, supervision of the Leucegene prognostic manuscript, validation of the Leucegene patient data, and editing of this manuscript. All authors revised the manuscript.

Data availability Most of the data generated or analyzed during this study are included in this published article and the Electronic Supplementary Material. The model that supports the findings of this study is available from Purple Squirrel Economics but restrictions apply to the availability of these data, which were used under license for the current study, and thus are not publicly available. Data are however available from the authors upon reasonable request and with permission of Purple Squirrel Economics.

\section{Compliance with Ethical Standards}

Funding This work was supported by the Government of Canada through Genome Canada and the Ministère de l'enseignement supérieur, de la recherche, de la science et de la technologie du Québec through Génome Québec to GS and JH. GS and JH are recipients of research chairs from the Canada Research Chair program and Industrielle-Alliance (Université de Montréal), respectively. The BCLQ is supported by grants from the Cancer Research Network of the Fonds de Recherche du Québec-Santé. Funding was provided to Adelphi Values for development of the model.

Conflict of interest Gabriel Tremblay, Ben Rousseau, Miriam Marquis, Cyrielle Beaubois, Guy Sauvageau, and Josée Hébert have no conflicts of interest that are directly relevant to the content of this article.

Open Access This article is distributed under the terms of the Creative Commons Attribution-NonCommercial 4.0 International License (http://creativecommons.org/licenses/by-nc/4.0/), which permits any noncommercial use, distribution, and reproduction in any medium, provided you give appropriate credit to the original author(s) and the source, provide a link to the Creative Commons license, and indicate if changes were made.

\section{References}

1. Statistics Canada. Table 103-0550: new cases of primary cancer (based on the July 2016 CCR tabulation file), by cancer type, age group and sex, Canada, provinces and territories. 2015. http://www5.statcan.gc.ca/cansim/a26?lang=eng\&id $=1030550$. Accessed Sep 2017.

2. Brandwein JM, Geddes M, Kassis J, et al. Treatment of older patients with acute myeloid leukemia (AML): a Canadian consensus. Am J Blood Res. 2013;3:141-64.

3. Schuh AC, Fletcher GG, Leber B, et al. Systemic treatment of acute myeloid leukemia (AML). Program in evidence-based care guideline No.: 12-9. 2016. https://www.cancercareontario.ca/sites /ccocancercare/files/guidelines/full/pebc12-9f.pdf. Accessed Nov 2018.

4. Cancer, Leukemia Group B, Farag SS, et al. Pretreatment cytogenetics add to other prognostic factors predicting complete remission and long-term outcome in patients 60 years of age or older with acute myeloid leukemia: results from Cancer and Leukemia Group B 8461. Blood. 2006;108:63-73.

5. Vey N, Coso D, Bardou VJ, et al. The benefit of induction chemotherapy in patients age $>$ or $=75$ years. Cancer. 2004;101:325-31.

6. Walter RB, Othus M, Borthakur G, et al. Prediction of early death after induction therapy for newly diagnosed acute myeloid leukemia with pretreatment risk scores: a novel paradigm for treatment assignment. J Clin Oncol. 2011;29:4417-23.

7. Grimwade D, Hills RK, Moorman AV, et al. Refinement of cytogenetic classification in acute myeloid leukemia: determination of prognostic significance of rare recurring chromosomal abnormalities among 5876 younger adult patients treated in the United Kingdom Medical Research Council trials. Blood. 2010;116:354-65.

8. Grimwade D, Walker H, Oliver F, et al. The importance of diagnostic cytogenetics on outcome in AML: analysis of 1,612 patients entered into the MRC AML 10 trial. The Medical Research Council Adult and Children's Leukaemia Working Parties. Blood. 1998;92:2322-33.

9. Rowe JM, Kim HT, Cassileth PA, et al. Adult patients with acute myeloid leukemia who achieve complete remission after 1 or 2 cycles of induction have a similar prognosis: a report on 1980 
patients registered to 6 studies conducted by the Eastern Cooperative Oncology Group. Cancer. 2010;116:5012-21.

10. Dohner H, Estey E, Grimwade D, et al. Diagnosis and management of AML in adults: 2017 ELN recommendations from an international expert panel. Blood. 2017;129:424-47.

11. Rockova V, Abbas S, Wouters BJ, et al. Risk stratification of intermediate-risk acute myeloid leukemia: integrative analysis of a multitude of gene mutation and gene expression markers. Blood. 2011;118:1069-76.

12. Mrozek K, Marcucci G, Nicolet D, et al. Prognostic significance of the European LeukemiaNet standardized system for reporting cytogenetic and molecular alterations in adults with acute myeloid leukemia. J Clin Oncol. 2012;30:4515-23.

13. Marquis M, Beaubois C, Lavallee VP, et al. High expression of HMGA2 independently predicts poor clinical outcomes in acute myeloid leukemia. Blood Cancer J. 2018;8:68.

14. Quebec Leukemia Cell Bank. http://bclq.org/. Accessed 22 Jul 2019.

15. Tremblay G, Haines P, Briggs A. A criterion-based approach for the systematic and transparent extrapolation of clinical trial survival data. J Health Econ Outcomes Res. 2014;2:147-60.

16. Latimer N. NICE DSU Technical Support Document 14: undertaking survival analysis for economic evaluations alongside clinical trials: extrapolation with patient-level data. 2011. http://www. nicedsu.org.uk. Accessed 22 Jul 2019.

17. Canadian Agency for Drugs and Technologies in Health. Guidelines for the economic evaluation of health technologies: Canada, 4th Edition. March 2017. https://www.cadth.ca/sites/default/files /pdf/guidelines_for_the_economic_evaluation_of_health_techn ologies_canada_4th_ed.pdf. Accessed Jun 2017.

18. Statistics Canada. Table 326-0021: consumer price index. 2016. http://www5.statcan.gc.ca/cansim/a26?lang=eng\&retrL ang=eng\&id=3260021\&\&pattern $=\&$ stByVal $=1 \& \mathrm{p} 1=1 \& \mathrm{p} 2=-$ $1 \&$ tabMode $=$ dataTable $\&$ csid. Accessed Nov 2018.

19. Uyl-de Groot CA, Gelderblom-den Hartog J, Huijgens PC, et al. Costs of diagnosis, treatment, and follow up of patients with acute myeloid leukemia in the Netherlands. J Hematother Stem Cell Res. 2001;10:187-92.

20. Reed SD, Anstrom KJ, Ludmer JA, et al. Cost-effectiveness of imatinib versus interferon-alpha plus low-dose cytarabine for patients with newly diagnosed chronic-phase chronic myeloid leukemia. Cancer. 2004;101:2574-83.

21. Ontario case costing initiative. CMG 610 Bone Marrow/Stem Cell Transpl; C920 Acute Myeloid Leukaemia. 2016.

22. Leunis A. The cost-effectiveness of personalized medicine strategies in acute myeloid leukemia. 2015. https://repub.eur.nl/ pub/78280/a.leunis.pdf. Accessed 22 Jul 2019

23. Unpublished Leucegene report. The Leucegene Project: Université de Montréal, 2019. https://leucegene.ca/. Accessed 22 Jul 2019.

24. Cressman S, Karsan A, Hogge DE, et al. Economic impact of genomic diagnostics for intermediate-risk acute myeloid leukaemia. Br J Haematol. 2016;174:526-35. 